\title{
Largest cliques in connected supermagic graphs
}

\author{
A. Lladó \\ Universitat Politècnica de Catalunya \\ Jordi Girona, 1, E-08034 Barcelona, Spain \\ allado@mat.upc.es
}

\begin{abstract}
A graph $G=(V, E)$ is said to be magic if there exists an integer labeling $f: V \cup E \longrightarrow[1,|V \cup E|]$ such that $f(x)+f(y)+f(x y)$ is constant for all edges $x y \in E$. Enomoto, Masuda and Nakamigawa proved that there are magic graphs of order at most $3 n^{2}+o\left(n^{2}\right)$ which contain a complete graph of order $n$. Bounds on Sidon sets show that the order of such a graph is at least $n^{2}+o\left(n^{2}\right)$. We close the gap between those two bounds by showing that, for any given graph $H$ of order $n$, there are connected magic graphs of order $n^{2}+o\left(n^{2}\right)$ containing $H$ as an induced subgraph. Moreover it can be required that the graph admits a supermagic labelling $f$, which satisfies the additional condition $f(V)=[1,|V|]$.
\end{abstract}

Keywords: Labelings of graphs, magic graphs, Sidon sets.

\section{Introduction}

A simple finite graph $G=(V, E)$ is said to be magic if there is a bijection $f: V \cup E \rightarrow[1,|V \cup E|]$ and a constant $k$ such that $f(x)+f(y)+f(x y)=k$ for each edge $x y \in E$. This notion was introduced by Kotzig and Rosa [8] in 1966 under the name of magic valuations. When $f(V)=[1,|V|]$ then the graph is supermagic; see for instance $[2,10]$. There are several related notions under the name of magic labellings; see the dynamic survey of Gallian [5].

An upper bound for the size of a magic graph containing a clique had been given already by Kotzig and Rosa [9], where they proved that, if $G=(V, E)$ is a magic graph containing a complete graph of order $n>8$, then

$$
|V|+|E| \geq n^{2}-5 n+14
$$

This result was improved by Enomoto, Masuda and Nakamigawa [3] to

$$
|V|+|E| \geq 2 n^{2}-O\left(n^{3 / 2}\right),
$$

by using the known bound for the size of a Sidon set given in ([4]).

\footnotetext{
${ }^{\dagger}$ Supported by the Ministry of Science and Technology of Spain, and the European Regional Development Fund (ERDF) under project-BFM-2002-00412 and by the Catalan Research Council under grant 2001SGR-00258.
}

1365-8050 (C) 2005 Discrete Mathematics and Theoretical Computer Science (DMTCS), Nancy, France 
Recall that a set $A$ of integers is said to be a Sidon set if all sums of pairs of elements (non necessarily different) of $A$, are pairwise distinct.

In 1941 Erdös and Turan [4] proved that a Sidon set $A \subset[1, N]$ always satisfies,

$$
|A| \leq N^{1 / 2}+N^{1 / 4}+1 .
$$

Kotzig [7] calls a set $A \subset \mathbb{Z}$ a well spread sequence if all sums of distinct elements in $A$ are pairwise different. He showed that, if $A \subset[1, N]$ with $N \geq 8$, then $N \geq 4+\left(\begin{array}{c}|A|-1 \\ 2\end{array}\right)$. Ruzsa [11] calls such a set a weak Sidon set. He gives a very nice short proof that a weak Sidon set in $[1, N]$ satisfies

$$
|A| \leq N^{1 / 2}+4 N^{1 / 4}+11 .
$$

If $A \subset V$ induces a clique in a magic graph $G=(V, E)$ with magic labelling $f$ then $f(A)$ is a weak Sidon set. That is, for each pair of vertices $x, y \in A$, we have $f(x)+f(y)=k-f(x y)$, so that the sums of labels of pairs of vertices in $A$ are pairwise distinct. Therefore $|A|$ is bounded by (3) with $N=|V \cup E|$, or $N=|V|$ if $f$ is super magic.

We want to point out that in 1972 Kotzig using well spread sequences proved in a long paper that $K_{n}$ is not magic for $n \geq 7$, [8]. The same result was reproved in 1999 by Craft and Tesar [1]. Note that inequality (3) shows directly this result for $n$ large enough.

There are explicit constructions of (weak) Sidon sets whose cardinality is close to the upper bound in (3). For instance, for any prime $p$, Singer gives a construction of a Sidon set of cardinality $p+1$ in $[1, N]$ with $N=p^{2}+p+1$ and Bose gives one of cardinality $p$ with $N=p^{2}-1$; see for instance [6]. Ruzsa [11] gives also such a construction of a Sidon set with $p-1$ elements in $\left[1, p^{2}-p\right]$. Since for each positive integer $n$ there is a prime $p$ such that $p \leq n+o(n)$, these constructions provide a Sidon set of order $n$ in $[1, N]$ with $N \leq n^{2}+o\left(n^{2}\right)$; see for instance [3, Lemma 3].

The existence of dense Sidon sets provide the means to obtain lower bounds for the largest possible clique in a connected magic graph. By using the construction of Singer [12] for dense difference sets Enomoto, Masuda and Nakamigawa [3] show that, for any graph $H$ with $n$ vertices and $m$ edges, there is a connected supermagic graph $G$ which contains $H$ as an induced subgraph such that

$$
|V(G)| \leq 2 m+2 n^{2}+o\left(n^{2}\right) .
$$

In particular, there are supermagic graphs $G$ containing the complete graph $K_{n}$, such that

$$
|V(G)| \leq 3 n^{2}+o\left(n^{2}\right) .
$$

On the other hand, if $G$ is a supermagic graph which contains a clique of order $n$, then (1) becomes

$$
|V(G)| \geq n^{2}-O\left(n^{3 / 2}\right),
$$

so that there is a gap between these upper and lower bounds. Our main result, which closes the gap between the bounds (5) and (6), is the following.

Theorem 1 Let $s(n)$ denote the minimum order of a connected supermagic graph containing a clique of order $n$. Then

$$
s(n)=n^{2}+o\left(n^{2}\right) .
$$

The proof of Theorem 1 can be adapted to show the following improvement of inequality (4).

Theorem 2 For any graph $H$ with $n$ vertices there is a connected supermagic graph $G$ of order $N=$ $n^{2}+o\left(n^{2}\right)$ which contains $H$ as an induced subgraph. 


\section{Magic graphs from Sidon sets}

We have already mentioned that the existence of a connected supermagic graph of order $N$ containing a clique of order $n$ implies the existence of a weak Sidon set of order $n$ in $[1, N]$. We will show that these two facts are actually equivalent.

Recall that a set of positive integers $A$ is a weak Sidon set if for any diferent elements $x, y, u, v \in A$, $x+y \neq u+v$. This is equivalent to say that $x-u \neq v-y$.

We first give a bound for a weak Sidon set $A$ which is good for any $|A| \geq 3$. The proof is similar to Ruzsa [11, Theorem 4.7].

Lemma 1 Let $A \subset[1, N]$ be a weak Sidon set with $|A| \geq 3$. Then

$$
N \geq \frac{|A|(|A|-3)}{2}+3+\epsilon(|A|)
$$

where $\epsilon(n)=1$ for $n \geq 6$ and $\epsilon(n)=0$ otherwise.

The following easy Lemma gives a simple criteria for a vertex labeling to extend to a super magic labeling.

Lemma 2 Let $G=(V, E)$ be a graph of order $n$ and $f: V \rightarrow[1, n]$ a bijection. Suppose that the edge sums of $f,\{f(x))+f(y), x y \in E\}$, form a consecutive set of integers. Then $f$ can be extended to a supermagic labeling of $G$.

Lemma 3 Let $G=(V, E)$ be a supermagic connected graph of order $N$. For each $N^{\prime}>N$ there is a supermagic connected graph $G^{\prime}$ which contains $G$ as an induced subgraph.

Lemma 4 Let $G=(V, E)$ be a graph of order $N$ and $f: V \rightarrow[1, N]$ a bijection such that the edge sums $f(x)+f(y), x y \in E$ are pairwise different. Then there is a super magic graph $G^{\prime}$ of order $N$ which contains $G$ as a spanning subgraph.

We are now ready for the proof of our main result.

Theorem 3 There is a connected super magic graph of order $N$ containing a clique of order $n$ if and only if there is a weak Sidon set $A \subset[1, N]$ of cardinality $n$.

Theorem 1 follows from Theorem 3 and the bounds described in the Introduction. Let $s(n)$ denote the minimum order of a supermagic graph containing a clique of order $n$. By (3) we have $s(n) \geq n^{2}+o\left(n^{2}\right)$. On the other hand, the known constructions of dense Sidon sets together with results on the distribution of primes provide in particular weak Sidon sets of cardinality $n$ in $[1, N]$ with $N=n^{2}+o\left(n^{2}\right)$, see for instance [3, Lemma 3]. By Theorem 3 we then have $s(n) \leq n^{2}+o\left(n^{2}\right)$.

Theorem 1 can be extended to construct connected supermagic graphs which contain any given graph $H$ as induced subgraph.

Corollary 1 Let $H$ be a connected graph of order $n$. If there is a weak Sidon set $A \subset[1, N]$ of cardinality $n$ then there exists a connected super magic graph $G$ of order $N$ which contains $H$ as an induced subgraph.

As a result of Corollary 1, there are connected supermagic graphs of order $N \leq n^{2}+o\left(n^{2}\right)$ which contain a given graph $H$ of order $n$ as an induced graph. This proves Theorem 2. 


\section{References}

[1] D. Craft, E. H. Tesar, On a question of Erdős about edge-magic graphs, Disc. Math. 207 (1999) 271-276.

[2] H. Enomoto, A.S. Lladó, T. Nakamigawa, G. Ringel, Super edge-magic graphs, SUT J. Math. 34 (1998) 105-109.

[3] H. Enomoto, K. Masuda, T. Nakamigawa, Induced Graph Theorem on Magic Valuations, Ars Combin. 56 (2000), 25-32.

[4] P. Erdôs, P. Turán, On a problem of Sidon in additive number theory and some related problems, J. of london Math. Soc. 16 (1941), 212-215.

[5] J.A. Gallian, A Dynamic Survey on Graph Labeling, The Electronic Journal of Combinatorics, DS6 (2000).

[6] H. Halberstam, K.F. Roth, Sequences, Springer-Verlag, New York, 1983.

[7] A. Kotzig, On well spread sets of integers, Publications du CRM-161, (1972) (83 pages)

[8] A. Kotzig, A. Rosa, Magic valuations of finite graphs, Canadian Mathematical Bulletin 13 (4) (1970), 451-461.

[9] A. Kotzig, A. Rosa, Magic Valuations of Complete Graphs, Publications du CRM-175, (1972) (8 pages)

[10] G. Ringel, A.S. Lladó, Another Tree Conjecture, Bull. Inst. Combin. Appl. 18 (1996), 83-85.

[11] I. Z. Ruzsa, Solving a linear equation in a set of integers, Acta Arithmetica LXV.3 (1993) 259-282.

[12] J. Singer, A theorem in finite projective geometry and some applications to number teory, Trans. Am. Math. Soc. 43 (1938), 377-385. 